\title{
High-Risk Smoking Behaviors and Barriers to Smoking Cessation Among Homeless Individuals
}

\author{
Joseph S Chen MD, Austin Huy Nguyen, Mark A Malesker PharmD, and Lee E Morrow MD MSc
}

\begin{abstract}
BACKGROUND: Although tobacco practices and the effects of tobacco use among the general American population are well described, minimal data exist regarding tobacco use and barriers to smoking cessation among homeless individuals. METHODS: Anonymous, voluntary surveys based on a previously implemented instrument were completed by 100 smoking individuals residing at a homeless shelter. These surveys assessed high-risk smoking behaviors and respondents' perceived barriers to long-term smoking cessation. RESULTS: Ninety percent of study participants reported engaging in at least one of the high-risk tobacco practices. Nicotine replacement therapy was perceived by respondents to be the most desired form of smoking cessation aid. Excessive stress with use of tobacco smoking to alleviate stress and anxiety was the most significant self-perceived barrier to smoking cessation. CONCLUSIONS: High-risk tobacco practices are remarkably common among smoking homeless individuals. Despite literature consistently showing that non-nicotine tobacco cessation pharmacotherapies (varenicline, buproprion) have higher smoking cessation rates, nicotine replacement monotherapy was perceived as more valuable by survey respondents. Although lack of financial resources was expected to be the biggest barrier to successful cessation, social stressors and the use of smoking to cope with homelessness were perceived as a greater obstacle in this cohort. Given the paucity of data on the long-term effects of the high-risk tobacco behaviors reported by these homeless smokers, this study highlights the need for further investigations regarding tobacco use and tobacco cessation in this vulnerable population. Key words: tobacco; smoking; tobacco use cessation; smoking cessation; homeless persons; health surveys; tobacco use disorder. [Respir Care 2016;61(5):640-645. (C) 2016 Daedalus Enterprises]
\end{abstract}

\section{Introduction}

Individuals who are homeless are $>3$ times as likely to smoke as individuals who are not homeless: Approximately 7 in 10 homeless adults smoke compared with 2 in 10

The authors are affiliated with the Creighton University School of Medicine, Omaha, Nebraska. Dr Morrow is also affiliated with the NebraskaWestern Iowa Veterans Affairs Medical Center, Omaha, Nebraska.

Dr Morrow presented a version of this paper at the CHEST Annual Meeting 2014, held October 24-28, 2014, in Austin, Texas.

The authors have disclosed no conflicts of interest.

Correspondence: Lee E Morrow MD MSc, Division of Pulmonary, Critical Care, and Sleep Medicine, Creighton University Medical Center, 601 North 30th Street, Suite 3820, Omaha, NE 68131. E-mail: lmorrow@creighton.edu.

DOI: $10.4187 /$ respcare.04439 adults in the general United States population. ${ }^{1-4}$ Aside from the inherent risks of life on the streets, this smoking discrepancy represents an additional health disparity for the homeless. Although most homeless individuals view smoking as a relatively harmless coping mechanism, many engage in unique risky smoking behaviors that potentially increase the harms associated with smoking. Furthermore, although many homeless individuals view their smoking as a temporary necessity that will abate when their homelessness resolves, multiple studies have demonstrated lower long-term cessation rates in this group of individuals. ${ }^{5-8}$

The general health risks associated with cigarette smoking are well established and include accelerated cardiovascular disease, a host of pulmonary diseases, and multiple cancers, among others. The attributable risk of smoking is further influenced by higher-risk smoking practices, such as heavy tobacco consumption and/or the use of non-filtered cigarettes. Among homeless individuals, there are a variety of unique high-risk smoking behaviors that are 
poorly described and that have unknown consequences. A survey study conducted on the streets of Los Angeles in $1993^{5}$ documented high rates of cigarette sharing, smoking discarded cigarette butts (commonly referred to as 'sniping'), smoking cigarettes remade from multiple discarded cigarette butts and/or filters, smoking cigarettes remade by others, blocking filter vents, and adding drugs to remade cigarettes. These practices are of particular interest because each may increase the potential for self-harm due to increased risk of toxin exposure, transmission of infectious disease, and/or higher overall consumption.

Although there is a robust literature base regarding theoretic limitations to smoking cessation in the homeless (ie, comorbid psychiatric disease, substance abuse, resource availability, noncompliance), there is a paucity of data focusing on barriers to tobacco cessation as perceived by homeless individuals themselves. As such, the present study seeks to, first, describe high-risk tobacco use practices in homeless people. Second, we seek to characterize the realworld barriers to smoking cessation that this population may encounter. Only through improved understanding of these issues will we achieve future platforms of change to better combat tobacco use among this vulnerable population.

\section{Methods}

Before initiating this study, the protocol and survey tools were reviewed and approved by the Creighton University institutional review board and the board of directors of the Siena/Francis House. This study was conducted in accordance with the amended Declaration of Helsinki, and verbal informed consent was obtained from all subjects. The Siena/Francis House is Nebraska's largest homeless shelter and is home of the Magis Clinic, a free medical clinic serving regional homeless and underinsured individuals. Participants were recruited from the general population of the Siena/Francis House and consisted of 100 consecutive homeless individuals willing to complete the anonymous questionnaire between March 1, 2014, and May 30, 2014. Participants were required to be at least $19 \mathrm{y}$ of age (the age of majority in Nebraska) and to have documentation of homelessness.

The survey tool was a questionnaire from a previous survey study by Aloot et $\mathrm{al}^{5}$ and more recently used in a study of homeless youth. ${ }^{9}$ The survey consisted of 22 questions requiring fill-in-the-blank responses or ranked responses using a Likert scale. Although this instrument is not copyrighted, consent to use the survey questions was obtained from the original authors. The survey was read aloud to all participants to overcome barriers imposed by illiteracy. The participants' responses were collected directly by the investigators to ensure accuracy and completeness. Individuals were not compensated for their par-

\section{QUICK LOOK}

\section{Current knowledge}

Homeless individuals are far more likely to smoke than adults in the general United States population. Although the general health risks of cigarette smoking are well known, they may be compounded by the unique highrisk smoking behaviors practiced by homeless individuals. These practices include cigarette sharing, smoking discarded cigarette butts, and modifying or adding drugs to cigarettes.

\section{What this paper contributes to our knowledge}

High-risk tobacco practices are remarkably common among homeless individuals who smoke. Nearly all of the participants surveyed in this study reported engaging in at least one of the high-risk tobacco practices. Relief of stress and anxiety by use of cigarettes is the leading reported reason for homeless individuals to continue smoking. When asked about cessation, nicotine replacement therapy was the most commonly desired method.

ticipation. After completion of the survey, participants were provided the opportunity to discuss their tobacco use and options for smoking cessation with one of the Magis Clinic's health-care providers.

Data were compiled using Excel 14.4.1 (Microsoft Corporation, Redmond, Washington) and then transferred to SPSS 21.0 for Windows (IBM Corporation, Armonk, New York) for statistical analysis. Only descriptive analyses (group mean, median, and SD) were performed given the nature of the study's goals. To numerically quantify and analyze respondents' perceptions of the utility of available smoking cessation tools, the 5-value Likert scale questions were assigned point values from 1 to 5 with larger numbers representing more favorable responses. Responses to the options in the Likert scale questions are displayed as a mean value (to summarize the overall perceived importance of any modality) as well as a histogram of the relative ranking of the various options. This was done because 2 options may ultimately have similar mean scores on the Likert-scale despite very different distributions of the underlying responses.

\section{Results}

The demographic characteristics and social descriptors are summarized in Table 1. The reported values are consistent with the population served by the Siena/Francis House. A summary of self-reported high-risk tobacco prac- 


\section{Smoking Behaviors and Barriers to Cessation in Homeless People}

Table 1. Self-Reported Demographics and Social Descriptors for the Homeless Population Studied $(N=100)$

\begin{tabular}{lc}
\hline \multicolumn{1}{c}{ Characteristics } & Values \\
\hline Male sex, $n(\%)$ & $78(78.0)$ \\
Age, mean \pm SD y & $45.4 \pm 10.9$ \\
Ethnicity, $n(\%)$ & \\
$\quad$ White & $68(68.0)$ \\
Black & $25(25.0)$ \\
Hispanic & $3(3.0)$ \\
Native American & $3(3.0)$ \\
Duration of homelessness, mean \pm SD mos & $32.1 \pm 52.8$ \\
Current substance abuse, $n(\%) *$ & $37(37)$ \\
Marijuana & $14(37.8)$ \\
Cocaine & $11(29.7)$ \\
Methamphetamine & $16(43.3)$ \\
Alcohol & $10(27.0)$ \\
Heroin & $3(8.1)$ \\
PCP & $1(2.7)$ \\
Age started smoking, mean \pm SD y & $15.0 \pm 6.0$ \\
Family smoking history, $n(\%) \dagger$ & \\
$\geq 1$ family member smokes & $88(88.0)$ \\
2 family members smoke & $32(36.3)$ \\
3 family members smoke & $35(39.7)$ \\
Father smokes & $63(63.0)$ \\
Mother smokes & $61(61.0)$ \\
Sibling smokes & $66(66.0)$ \\
& \\
$*$ Some participants reported multiple substances used along with tobacco; thus, summation of \\
collected percentages may be $>100 \%$. \\
$\dagger$ Some participants may have listed multiple family members that used tobacco; thus, \\
summation of collected percentages may be $>100 \%$. \\
\hline
\end{tabular}

tices is shown in Table 2. Overall, only $10.0 \%$ of study participants did not participate in any of the high-risk behaviors listed in the survey. Although $86.0 \%$ of participants had been advised at least once to quit tobacco, only $25 \%$ of these individuals had ever asked for assistance with smoking cessation (Table 3).

Regarding the perceived efficacy of the smoking cessation choices offered as monotherapy, nicotine replacement monotherapy received the highest mean Likert score overall (3.37 of a possible 5.00 points). This form of therapy was followed by non-nicotine-containing medications, such as varenicline or bupropion (3.06 points), no therapy (2.70 points), support from family and/or friends (2.56 points), and formal smoking cessation counseling (2.21 points). In addition to having the highest average score, the distribution of responses (Fig. 1) showed that nicotine replacement monotherapy was the agent most frequently chosen as having the highest likelihood of facilitating cessation $(35 \%)$. The frequency with which other agents were chosen as being most efficacious was: no therapy (27\%), nonnicotine-containing medications $(23 \%)$, family support $(10 \%)$, and formal counseling (5\%).
Table 2. Self-Reported Smoking Practices, Including High-Risk Behaviors $(N=100)$

\begin{tabular}{lc}
\hline \hline \multicolumn{1}{c}{ Practices } & Values \\
\hline $\begin{array}{c}\text { Cigarette consumption, mean } \pm \mathrm{SD} \\
\text { (range) packs/d }\end{array}$ & $1.2 \pm 2.2(0.1-20)$ \\
Time to first cigarette each morning, & \\
mean \pm SD (range) min & $36.7 \pm 64.3(0-500)$ \\
Frequency of talking about smoking, & \\
mean \pm SD (range) times/d & $5.5 \pm 14.7(0-100)$ \\
High-risk smoking practices, $n(\%) *$ & \\
Cigarette sharing & \\
Cigarette remaking & $73(73.0)$ \\
Smoking discarded butts & $37(37.0)$ \\
Smoking discarded filters & $34(34.0)$ \\
Altering cigarette filter or vents & $10(10.0)$ \\
Removing tobacco and using cigarette & $32(32.0)$ \\
to smoke drugs & $36(36.0)$ \\
Marijuana & \\
Cocaine & $35(97.2)$ \\
Adding drugs to cigarette tobacco & $2(5.6)$ \\
Marijuana & $25(25.0)$ \\
Cocaine & $15(60.0)$ \\
Methamphetamine & $8(32.0)$ \\
PCP & $2(8.0)$ \\
\end{tabular}

* Multiple high-risk practices may be reported by participants; thus, summation of collected percentages may be $>100 \%$.

Table 3. Self-Reported History of Smoking Cessation Attempts for the Homeless Population Studied $(N=100)$

\begin{tabular}{lc}
\hline \hline \multicolumn{1}{c}{ Parameters } & Values \\
\hline Anyone advised cessation, $n(\%)^{*}$ & $86(86.0)$ \\
Doctor & $68(79.1)$ \\
Family & $52(60.4)$ \\
Friends & $31(36.0)$ \\
Other & $3(3.5)$ \\
Feel stopping is important, $n(\%)$ & $51(51.0)$ \\
Have requested cessation help, $n(\%)$ & $25(25.0)$ \\
Attempted cessation past year, $n(\%)$ & $40(40.0)$ \\
1 attempt & $15(37.5)$ \\
2 attempts & $14(35.0)$ \\
$3-5$ attempts & $8(20.0)$ \\
$>5$ attempts & $3(7.5)$ \\
Cessation attempts past year, mean \pm SD & $2.4 \pm 1.6$ \\
Longest cessation period past year, mean \pm SD d & $55.0 \pm 159.3$ \\
& \\
* Some participants may have reported multiple individuals who have previously advised them \\
in tobacco cessation; thus, summation of collected percentages may be $>100 \%$. \\
\hline
\end{tabular}

Among the subject-perceived barriers to successful smoking cessation, excessive stress and use of cigarettes to relieve anxiety received the highest mean Likert score overall (3.95 of a possible 5.00 points), followed by cravings ( 3.45 points), lack of resources for 


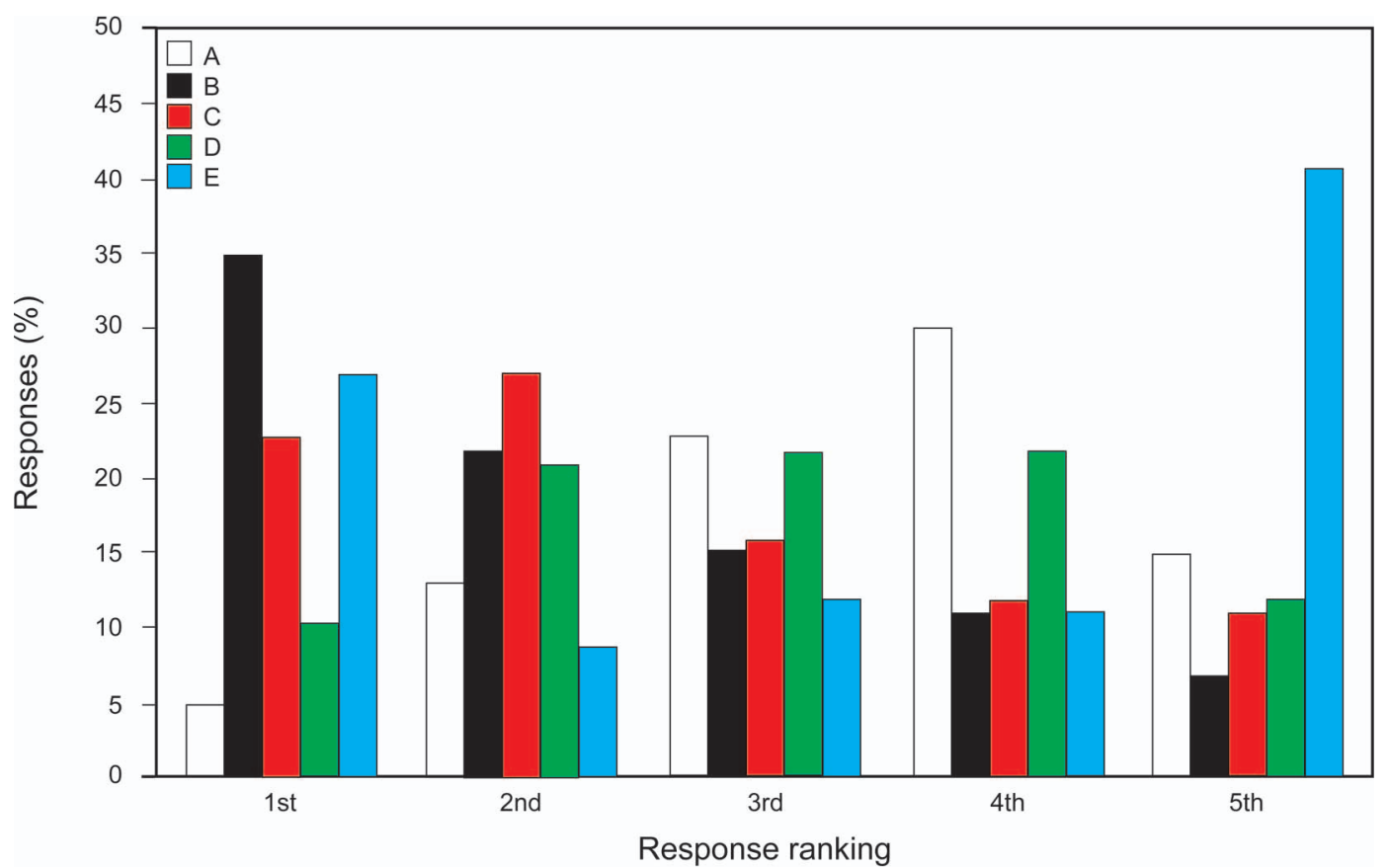

Fig. 1. Distribution of responses for tobacco cessation method. Shown are survey responses to the question: What do you think would help you to stop smoking? with the following response options: $A=$ smoking cessation counseling programs; $B=$ nicotine replacement therapy; $\mathrm{C}=$ non-nicotine-containing medication (eg, varenicline, buproprion); $\mathrm{D}=$ support from friends and/or family; $E=$ nothing.

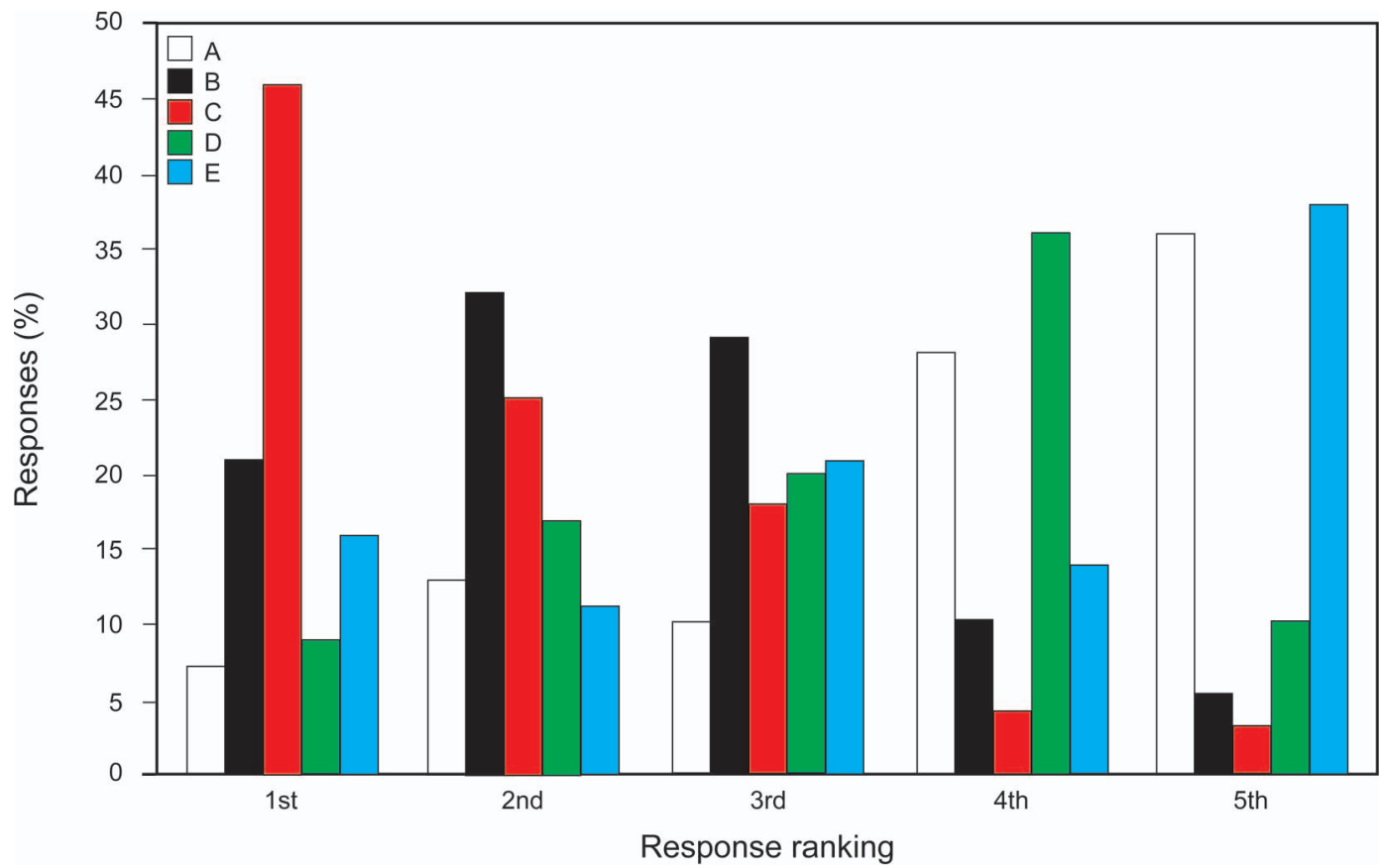

Fig. 2. Distribution of responses for barriers to cessation. Shown are survey responses to the question: What barriers prevent you from stopping smoking? with the following response options: $\mathrm{A}=$ peer pressure; $\mathrm{B}=$ cravings; $\mathrm{C}=$ too much stress and cigarettes used to relieve anxiety; $\mathrm{D}$ = lack of resources (eg, money for medication or counseling); $\mathrm{E}=$ not an important goal.

cessation programs/support (2.55 points), not viewing cessation as an important goal (2.53 points), and peer pressure (2.09 points). In addition to having the highest average score, the distribution of responses (Fig. 2) showed that stress was the barrier most frequently chosen as being most likely to impede cessation (46\%). The frequency with which other barriers were chosen as being the biggest obstacle was: cravings (21\%), lack of 
perceived importance of cessation (16\%), lack of resources $(9 \%)$, and peer pressure $(7 \%)$.

\section{Discussion}

In this study, we aimed to describe the variety and prevalence of high-risk smoking behaviors among homeless individuals. We found that high-risk smoking behaviors were remarkably common, seen in $90 \%$ of survey respondents, and that most participants engaged in more than one such activity. Although some of these behaviors appear relatively benign (ie, sharing cigarettes), others carry significant potential risk (ie, replacing cigarette tobacco with drugs or adding drugs to the tobacco). Because the longterm implications of these actions have not been rigorously studied, the long-term consequences of these actions remain unknown.

We also attempted to better understand the perceptions of the homeless regarding quitting smoking and barriers to successful cessation. The percentage of smokers in this study who stated that they wanted to quit $(51.0 \%)$ was lower than the frequently cited value in the general population $(59.1 \%)$, whereas the percentage of homeless smokers who tried to quit in the past year $(40.0 \%)$ was also lower than that of the general population $(51.7 \%){ }^{8}$

From the perspective of our homeless participants, smoking cessation using nicotine replacement monotherapy was most valued, since it received the highest mean score and was most frequently ranked first out of the 5 choices. Interestingly, non-nicotine-containing pharmacotherapy (varenicline or buproprion) was viewed on par with no therapy (quitting outright): Although non-nicotinecontaining pharmacotherapy had a slightly higher mean Likert score, quitting without assistance was chosen more frequently as the most preferred option. Support from family/friends and formal smoking cessation counseling were consistently viewed as least helpful, receiving both the lowest mean Likert scores and the lowest frequency of selection as the favored option.

These perceptions merit further evaluation, given their disconnect from data showing that non-nicotine-containing pharmacotherapy has the highest successful cessation rates, followed by nicotine replacement monotherapy and counseling, all of which are superior to no therapy. ${ }^{8} \mathrm{We}$ speculate that the lower priority given to family support and formal counseling may stem from prior exhaustion and/or failure of family support networks and other counseling programs in other aspects of these individuals' complicated lives. We also question whether quitting without assistance was perceived as an appealing strategy for respondents because this provides the greatest sense of empowerment during a time where many have lost control over various aspects of their lives. Other possible explanations for the relatively high rankings of nicotine replace- ment therapy and quitting outright include their familiarity (given over-the-counter availability) or mistrust of the medical establishment in this population.

Somewhat surprisingly, stress was consistently viewed as the biggest barrier to smoking cessation and was closely followed by nicotine cravings, regardless of whether the mean Likert score or frequency of selection as the most significant barrier was used as the comparison metric. Availability of cessation resources and perceived lack of importance were generally viewed on par with one another: resource availability had a slightly higher mean Likert score, whereas lack of importance was more frequently chosen as the largest barrier. Peer pressure was clearly the least important barrier.

Given the limited monetary resources of our target population, we anticipated that lack of resources would be the biggest perceived barrier to smoking cessation. However, the stress of coping with homelessness is a bigger obstacle for the homeless, superseding even nicotine cravings. We suspect that the perceived lack of importance of smoking cessation reported is probably due to the relative importance when compared with other obstacles in these participants' lives (homelessness, drug use, legal concerns, psychiatric illness, etc).

The results of this study may also be of particular relevance to respiratory therapy in the emergency room. Use of the emergency room by homeless individuals for their primary care creates a need for emergency care professionals to be particularly attentive to these issues. Emergency room respiratory therapists should play a central role in exploring the use of high-risk behaviors and barriers to cessation. Furthermore, the emergency room therapist is similarly important in educating homeless individuals regarding the health hazards of such high-risk behaviors and for helping patients to overcome their unique cessation barriers.

Although our study is unique in its design and scope, and is the largest study done to date in this arena using a previously implemented survey tool, it has several limitations that merit consideration. First, this was a singlecenter study wherein participation was voluntary. Accordingly, our results may not be entirely generalizable. However, nonparticipation rates were very low (estimated at $<5 \%$ ), and respondents were incredibly generous with their time and opinions. Also, the convenience sampling of volunteers may make this study prone to self-selection bias. Second, all of the measured variables are subjective and self-reported. Given known associations between homelessness, psychiatric disease, and substance abuse, it is possible that some responses are inaccurate. Third, the sex distribution of our respondents shows a higher male representation (78\%) relative to the most recent census data, wherein $70.4 \%$ of this state's homeless population was male. ${ }^{10}$ There was a similar slight disconnect between 
the reported racial breakdown of our respondents and the general homeless population in Nebraska. These discrepancies are possibly manifestations of our relatively small sample size $(N=100)$. Last, within the homeless community, there are different degrees of homelessness. These range from those who are completely impoverished and consistently reside in shelters, to those with some resources who intermittently live outside the shelter short-term, to those whose finances require them to use some shelterbased resources while allowing them to live outside the shelter (the so-called car homeless population). Differential participation by these subgroups of homeless individuals may affect our reported observations.

\section{Conclusions}

Given the current paucity of data examining homelessness and patterns of tobacco use, high-risk smoking behaviors, long-term sequelae of such behavior, attitudes regarding smoking cessation, and the attendant costs to the health-care system, this study has provided the initial foundation for multiple avenues of future investigation. Only through a better understanding of the complex interplay of smoking behavior, education, substance abuse, and psychosocial factors will we succeed in reducing tobacco abuse in this vulnerable population.

\section{ACKNOWLEDGMENTS}

We thank the Siena Francis House Board of Directors and the Creighton University School of Medicine's Magis Health Clinic for allowing in- vestigators to conduct interviews during clinic hours. In addition, we are incredibly grateful to the many individuals who were so gracious in giving time to participate in the present study.

\section{REFERENCES}

1. Arnsten JH, Reid K, Bierer M, Rigotti N. Smoking behavior and interest in quitting among homeless smokers. Addict Behav 2004; 29(6):1155-1161.

2. Connor SE, Cook RL, Herbert MI, Neal SM, Williams JT. Smoking cessation in a homeless population: there is a will, but is there a way? J Gen Intern Med 2002;17(5):369-372.

3. Okuyemi KS, Caldwell AR, Thomas JL, Born W, Richter KP, Nollen $\mathrm{N}$, et al. Homelessness and smoking cessation: insights from focus groups. Nicotine Tob Res 2006;8(2):287-296.

4. Snyder LD, Eisner MD. Obstructive lung disease among the urban homeless. Chest 2004;125(5):1719-1725.

5. Aloot CB, Vredevoe DL, Brecht ML. Evaluation of high-risk smoking practices used. Cancer Nurs 1993;16(2):123-130.

6. Okuyemi KS, Thomas JL, Hall S, Nollen NL, Richter KP, Jeffries SK, et al. Smoking cessation in homeless populations: a pilot clinical trial. Nicotine Tob Res 2006;8(5):689-699.

7. American Lung Association Research and Program Services Epidemiology and Statistics Unit. Trends in tobacco use. American Lung Association; 2011. http://www.lung.org/assets/documents/research/ tobacco-trend-report.pdf. Accessed December 2, 2015.

8. Spector A, Alpert H, Karam-Hage M. Smoking cessation delivered by medical students is helpful to homeless population. Acad Psychiatry 2007;31(5):402-405.

9. Tucker JS, Shadel WG, Golinelli D, Mullins L, Ewing B. Sniping and other high-risk smoking practices among homeless youth. Drug Alcohol Depend 2015;154:105-110.

10. Smith AS, Holmberg C, Jones-Puthoff M. The emergency and transitional shelter population: 2010. https://www.census.gov/prod/ cen2010/reports/c2010sr-02.pdf. Accessed December 2, 2015. 